\title{
Updates in the Management of CLL/SLL: Sequencing Therapy and the Role of Minimal Residual Disease Testing
}

\author{
Presented by William G. Wierda, MD, PhD
}

\begin{abstract}
Progress in the treatment of chronic lymphocytic leukemia/small lymphocytic lymphoma has led to a renewed focus on minimal residual disease as a treatment endpoint, particularly in assessing survival outcomes with combination therapies. B-cell receptor signaling pathway inhibitor monotherapy is associated with low rates of undetectable minimal residual disease, and continued treatment is still required; however, $\mathrm{BCL}-2$ inhibitor therapy with venetoclax is associated with high rates of undetectable minimal residual disease, as are new combinations with BCL-2 inhibitors. Early research indicates that BCL-2 inhibitors may be an effective treatment option for disease that is refractory to Bruton's tyrosine kinase (BTK) inhibitor therapy.
\end{abstract}

Evolving standard first-line approaches to the treatment for chronic lymphocytic leukemia/small lymphocytic lymphoma (CLL/SLL) are once again focusing on the integration of minimal residual disease (MRD) status as a response assessment tool. MRD status correlates with clinical outcomes, therefore making it a good early indicator of response and expected outcomes, noted William G. Wierda, MD, PhD, Professor of Medicine, The University of Texas MD Anderson Cancer Center, at the NCCN 2020 Virtual Congress: Hematologic Malignancies.

"I think it's important to highlight where we're moving with treatment, as well as our goals of treatment, because they're changing," Dr. Wierda said. "We have more and more effective treatments available, and we're learning how to better use those drugs together."

According to Dr. Wierda, goals in the management of CLL/SLL start with eliminating chemoimmunotherapy altogether and replacing it with more effective combinations of small molecule inhibitors. "We'd also like to get as high a proportion of patients in as deep a remission as possible with a fixed duration of treatment (ie, increase the proportion of patients in complete remission [CR] with undetectable MRD with well-tolerated non-chemoimmunotherapy treatment) to improve and lengthen both progression-free survival (PFS) and overall survival (OS). We are also interested in how to manage patients with refractory disease, particularly disease that is refractory to Bruton's tyrosine kinase (BTK) inhibitors as well as BCL-2 inhibitors," he said.

In addition, "immune reconstitution is also a very important topic that we are focussing on, as patients with CLL do not have normal immune function and are at higher risk for infections and second cancers. This appears to be an emerging, more common problem in the CLL population, more so than managing the disease in some patients," he said.

\section{MRD Evaluation}

CLL is a multicompartmental disease (involving bone marrow, blood, lymph nodes, spleen, liver, and lymph nodes), but MRD is typically evaluated only in the blood or bone marrow. Flow cytometry is commonly used to measure MRD, but next-generation DNA sequencing (NGS)-based assays are now being more broadly applied. Recently, the FDA cleared an NGS-based assay for MRD (clonoSEQ).

In the German CLL Study Group analysis, MRD status at the end of chemoimmunotherapy treatment correlated with better survival in a multivariate analysis; patients experiencing undetectable MRD in their blood had longer PFS and OS." "We have looked at similar patient populations and treatment strategies in our work," Dr. Wierda said. "In patients who received fludarabine/cyclophosphamide/rituximab (FCR), those with a mutated immunoglobulin heavy-chain variable (IGHV) region gene had extended PFS beyond 10 years. Many of those patients are MRD-negative, and that's 


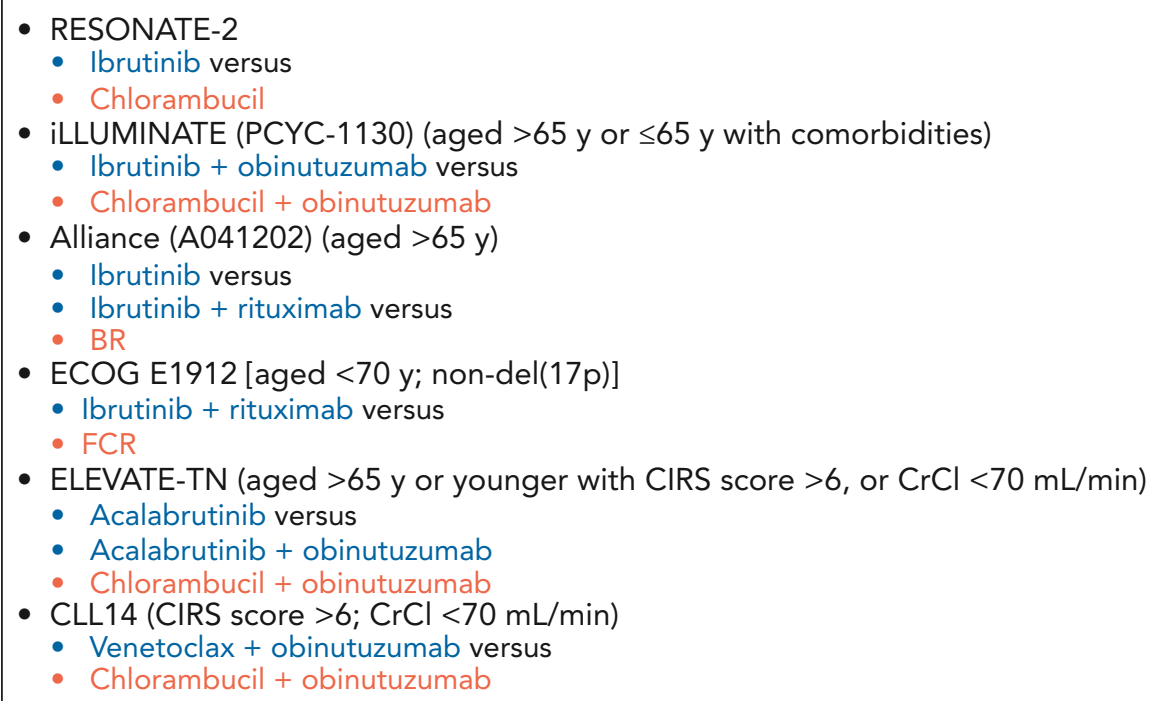

Figure 1. First-line phase III randomized trials. Regimens in orange represent the control arm. Regimens in blue were superior to orange regimens in the control arm.

Abbreviations: BR, bendamustine/rituximab; CIRS, Cumulative Illness Rating Scale; $\mathrm{CrCl}$, creatinine clearance; $\mathrm{FCR}$, fludarabine/cyclophosphamide/rituximab.

the population that we have proposed as potentially cured by chemoimmunotherapy. ${ }^{2 "}$ Patients with a mutated IGHV and an undetectable MRD at the end of 6 cycles of FCR have the best outcome in terms of PFS.

According to Dr. Wierda, although MRD has been studied in CLL for years (mainly with chemoimmunotherapy), this marker became less important with the use of BTK inhibitors because these inhibitors do not result in deep remissions. However, with the development of B-cell lymphoma 2 (BCL-2) inhibitors (eg, venetoclax) that induce deeper remissions in CLL, "MRD is becoming more and more important as we integrate venetoclax into the therapeutic landscape of CLL," he clarified.

\section{Selecting First-Line Treatment in CLL}

According to Dr. Wierda, results of 3 different tests are needed before starting a patient on first-line treatment for CLL.

"I need to know their fluorescence in situ hybridization (FISH) status, particularly with regard to $17 \mathrm{p}$ deletion; I need to know their TP53 mutation status; and I'm interested in knowing their IGHV mutation status," he said. "These 3 results help direct me in terms of recommending a first-line therapy."

He pointed out 5 recently published randomized phase III trials of BTK inhibitor-based therapy versus chemoimmunotherapy (Figure 1). ${ }^{3-7}$ These trials were performed in various patient populations and spanned the spectrum of intensity of chemoimmunotherapy, from chlorambucil monotherapy to more intensive regimens such as FCR. ${ }^{3-7}$ All of these studies have shown improved PFS with BTK inhibitor-based therapy (ibrutinib-based or acalabrutinib-based) over chemoimmunotherapy. The RESONATE- $2^{3}$ and ECOG E $1912^{6}$ trials also showed improvements in OS.

The randomized phase III CLL14 trial looked at venetoclax/obinutuzumab versus chlorambucil/ obinutuzumab in the frontline setting in a more frail population. This was also a positive study, he noted, showing improvement in PFS and deeper remissions for venetoclax-based therapy, with no difference in OS. At 18 months' follow-up, $47 \%$ of patients who received venetoclax were MRD-negative (measured by blood-based assay), compared with $7 \%$ who received chlorambucilbased treatment. ${ }^{8}$

Although ibrutinib was the first BTK inhibitor, others have been developed and approved. Ibrutinib inhibits BTK, but also several other kinases. "This is important, because if other kinases are inhibited, that may be leading to some of the other off-target toxicities that we see with ibrutinib," said Dr. Wierda. "Acalabrutinib inhibits BTK with fewer off-target kinases inhibited, which may contribute to the observation that there appears to be less toxicity associated with acalabrutinib."

Acalabrutinib was approved in the United States in the frontline settings, based on results from the ELEVATE-TN trial. ${ }^{7}$ In the ELEVATE-TN trial, acalabrutinib in combination with obinutuzumab showed a statistically significant and clinically meaningful improvement in PFS versus obinutuzumab/chlorambucil. PFS was also significantly improved with single-agent acalabrutinib versus obinutuzumab/chlorambucil.

"I think it's important that we obtain further followup with acalabrutinib," Dr. Wierda noted. "Acalabrutinib, 
like ibrutinib, is continuous and indefinite treatment, and I think there's still some controversy around whether or not the CD20 antibody, particularly obinutuzumab, adds to the benefits that we see with acalabrutinib in the front line."

To summarize first-line therapy options in CLL, Dr. Wierda recommends BTK inhibitor-based therapy for patients with $17 \mathrm{p}$ deletions. For patients without $17 \mathrm{p}$ deletions, age and comorbidities should be considered.

"If a patient has an unmutated $I G H V$, and access to the medical setting may be more challenging, then a BTK inhibitor may be a more favorable option," he said. "If they're able to get back and forth to the doctor and they have a mutated IGHV, I'm interested in BCL-2 inhibitor-based therapy (eg, venetoclax-based therapy)."

In the case of younger and fit patients with unmutated $I G H V$, "I like these patients to get a good deep remission, get off treatment and be off treatment for several years, and then retreat them when the disease relapses," Dr. Wierda explained. In patients with mutated $I G H V$, FCR should still be considered. "I'm optimistic that eventually that will be replaced by venetoclax-based therapy," he said. "But we need more follow-up to be secure in the statement that those patients are achieving a deep enough remission to come off treatment and potentially to be cured like we are seeing in about $50 \%$ of patients with unmutated $I G H V$ treated with FCR-based therapy."

\section{Combination Therapies With Venetoclax and BTK Inhibitor}

BTK inhibitor-based therapy does not typically lead to deep remissions; therefore, disease can still be measured even in patients who have been on ibrutinib or acalabrutinib for $>1$ year. Because BCL-2 inhibitor-based therapy with venetoclax is associated with high rates of undetectable MRD, an ongoing trial at MD Anderson is evaluating "venetoclax consolidation" in patients with measurable disease after taking ibrutinib for $\geq 1$ year. "In the 22 patients who reached a year of combination therapy with venetoclax and ibrutinib, $68 \%$ of them achieved undetectable MRD status in the bone marrow, ${ }^{9}$ " Dr. Wierda reported. "So we're excited about this study. We have expanded it, and we're still enrolling (ClinicalTrials.gov identifier: NCT02910583)."

According to Dr. Wierda, ibrutinib and venetoclax are clinically complementary, with nonoverlapping mechanisms of action and toxicity profiles. Venetoclax is highly effective at clearing blood and bone marrow disease, whereas ibrutinib is extremely effective at shrinking lymph nodes.

Confirmatory studies are now being performed with this drug combination. In the frontline CAPTIVATE trial of patients with CLL aged $<70$ years, $72 \%$ had undetectable MRD after 12 months of combination therapy with venetoclax/ibrutinib. ${ }^{10}$ Other combination studies are underway, and a novel triplet regimen containing acalabrutinib/venetoclax/obinutuzumab has shown high rates of undetectable MRD in the bone marrow: $75 \%$ after 16 cycles of treatment with the triplet. ${ }^{11}$

\section{Treatment of Relapsed/Refractory CLL}

"Our treatments for relapsed disease have largely been driven by the population of patients who have been enrolled in the trials for relapsed disease," Dr. Wierda said. "But the characteristics of those patients are shifting."

The phase III RESONATE trial showed improved outcomes with ibrutinib-based therapy over ofatumumabbased therapy. ${ }^{12}$ However, as is true in the frontline setting, $17 \mathrm{p}$ deletion is also a high-risk feature in the relapsed setting, and these patients had worse survival. "So we still worry about patients with 17 p deletion," he noted. Results of the ASCEND trial led to FDA approval of acalabrutinib for relapsed/refractory CLL. In this trial, acalabrutinib monotherapy was associated with improved PFS over rituximab/idelalisib or rituximab/ bendamustine. ${ }^{13}$

The phase III MURANO trial of venetoclax/rituximab in relapsed CLL showed high rates of MRD negativity versus bendamustine/rituximab. ${ }^{14}$ "Sixty percent of patients treated with venetoclax on this study achieved undetectable MRD status," Dr. Wierda said. "This is unexpectedly high for a relapsed treatment." In the case of refractory disease to BTK inhibitor therapy, resistance tends to be associated with BTKC481 mutation. ${ }^{15}$ Venetoclax has been studied in this setting and has shown activity in the patient population with CLL that is predominantly refractory to ibrutinib, he added. ${ }^{16}$

Dr. Wierda pointed out some exciting new data with LOXO-305, a reversible inhibitor of BTK. Early data from a small number of patients have shown activity in patients with refractory CLL or those previously treated with a BTK inhibitor. ${ }^{17}$ "This trial has continued to enroll, and we're looking forward to an update at ASH this year," he reported.

According to Dr. Wierda, much progress has been made in CLL, but there is still work to be done. "We're expecting to see very very long remissions and treatmentfree intervals with these new drug combinations," he added. "And I'm optimistic about the prospect of curing many of these patients."

Disclosures: Dr. Wierda has disclosed that he has received grant/research support from AbbVie, Inc., AstraZeneca Pharmaceuticals LP/Acerta Pharma Celgene Corporation/Juno Therapeutics, Inc., Cyclacel Pharmaceuticals, Inc. Genentech, Inc., Gilead Sciences, Inc., GlaxoSmithKline/Novartis Pharmaceuticals Corporation, Janssen Pharmaceutica Products, LP, Kite Pharma, Loxo Oncology, Inc., Oncternal Therapeutics, Inc., Pharmacylics, Sunesis Pharmaceuticals, Inc., and Xencor, Inc.

Correspondence: William G. Wierda, MD, PhD, The University of Texas MD Anderson Cancer Center, 1515 Holcombe Boulevard, Unit 428, Houston, TX 77030. Email: wwierda@mdanderson.org 


\section{References}

1. Kovacs G, Robrecht S, Fink AM et al. Minimal residual disease assessment improves prediction of outcome in patients with chronic lymphocytic leukemia (CLL) who achieve partial response: comprehensive analysis of two phase III studies of the German CLL Study Group. J Clin Oncol 2016; 34:3758-3765.

2. Thompson PA, Tam CS, O'Brien SM, et al. Fludarabine, cyclophosphamide, and rituximab treatment achieves long-term disease-free survival in IGHV-mutated chronic lymphocytic leukemia. Blood 2016; 127:303-309.

3. Barr P, Robak T, Owen CJ, et al. Updated efficacy and safety from the phase 3 RESONATE-2 study: ibrutinib as first-line treatment option in patients 65 years and older with chronic lymphocytic leukemia/small lymphocytic leukemia [abstract]. Blood 2016;128: Abstract 234.

4. Moreno C, Greil R, Demirkan F et al. Ibrutinib plus obinutuzumab versus chlorambucil plus obinutuzumab in first-line treatment of chronic lymphocytic leukaemia (iLLUMINATE): a multicentre, randomised, openlabel, phase 3 trial. Lancet Oncol 2019;20:43-56.

5. Woyach JA, Ruppert AS, Heerema NA, et al. Ibrutinib regimens versus chemoimmunotherapy in older patients with untreated CLL. N Engl J Med 2018;379:2517-2528.

6. Shanafelt TD, Wang $\mathrm{V}$, Kay NE, et al. A randomized phase III study of ibrutinib (PCl-32765)-based therapy vs. standard fludarabine, cyclophosphamide, and rituximab (FCR) chemoimmunotherapy in untreated younger patients with chronic lymphocytic leukemia (CLL): a trial of the ECOG-ACRIN Cancer Research Group (E1912) [abstract]. Blood 2018; 132:Abstract LBA-4.

7. Sharman JP, Egyed M, Jurczak W, et al. Acalabrutinib with or without obinutuzumab versus chlorambucil and obinutuzumab for treatmentnaive chronic lymphocytic leukaemia (ELEVATE TN): a randomised, controlled, phase 3 trial. Lancet 2020;395:1278-1291.

8. Fischer $\mathrm{K}, \mathrm{Al}$-Sawaf $\mathrm{O}$, Fink $\mathrm{A}$, et al. Venetoclax and obinutuzumab in chronic lymphocytic leukemia. Blood 2017;129:2702-2705.
9. Thompson PA, Keating MJ, Jain N, et al. Venetoclax added to ibrutinib in high-risk CLL achieves a high rate of undetectable minimal residual disease [abstract]. Blood 2019;134(Suppl):Abstract 358.

10. Siddiqi T, Tam CS, Allan JN, et al. First-line ibrutinib (IBR) + venetoclax (VEN) for patients (pts) with chronic lymphocytic leukemia (CLL)/small lymphocytic lymphoma (SLL): efficacy and safety results from CAPTIVATE MRD cohort. Presented at the 25th European Hematology Association Virtual Congress; June 11-14, 2020. Abstract S158.

11. Lampson BL, Tyekucheva $\mathrm{S}$, Crombie JL, et al. Preliminary safety and efficacy results from a phase 2 study of acalabrutinib, venetoclax and obinutuzumab in patients with previously untreated chronic lymphocytic leukemia (CLL) [abstract]. Blood 2019;134:Abstract 32.

12. Byrd JC, Brown JR, O'Brien S, et al. Ibrutinib versus ofatumumab in previously treated chronic lymphoid leukemia. N Engl J Med 2014;371:213-223.

13. Ghia P, Pluta A, Wach $M$, et al. Acalabrutinib (Acala) versus idelalisib plus rituximab (IdR) or bendamustine plus rituximab (BR) in relapsed/refractory $(\mathrm{R} / \mathrm{R})$ chronic lymphocytic leukemia (CLL): ASCEND final results [abstract] J Clin Oncol 2020;38:Abstract 8015.

14. Kater AP, Seymour JF, Hillmen $P$, et al. Fixed duration of venetoclaxrituximab in relapsed/refractory chronic lymphocytic leukemia eradicates minimal residual disease and prolongs survival: post-treatment follow-up of the MURANO phase III study. J Clin Oncol 2019;37:269-277.

15. Lampson BL, Brown JR. Are BTK and PLCG2 mutations necessary and sufficient for ibrutinib resistance in chronic lymphocytic leukemia? Expert Rev Hematol 2018;11:185-194

16. Jones JA, Mato AR, Wierda WG, et al. Venetoclax for chronic lymphocytic leukaemia progressing after ibrutinib: an interim analysis of a multicentre open-label, phase 2 trial. Lancet Oncol 2018;19:65-75.

17. Mato AR, Flinn IW, Pagel JM, et al. Results from a first-in-human, proof-ofconcept phase 1 trial in pretreated B-cell malignancies for Loxo-305, a next-generation, highly selective, non-covalent BTK inhibitor [abstract]. Blood 2019;134:Abstract 501 